PROCEEDINGS OF THE

AMERICAN MATHEMATICAL SOCIETY

Volume 131, Number 3, Pages 973-978

S 0002-9939(02)06572-3

Article electronically published on July 17, 2002

\title{
ON POLYNOMIAL PRODUCTS IN NILPOTENT AND SOLVABLE LIE GROUPS
}

\author{
KAREL DEKIMPE
}

(Communicated by Ronald A. Fintushel)

\begin{abstract}
We are dealing with Lie groups $G$ which are diffeomorphic to $\mathbb{R}^{n}$, for some $n$. After identifying $G$ with $\mathbb{R}^{n}$, the multiplication on $G$ can be seen as a map $\mu: \mathbb{R}^{n} \times \mathbb{R}^{n} \rightarrow \mathbb{R}^{n}:(\mathbf{x}, \mathbf{y}) \mapsto \mu(\mathbf{x}, \mathbf{y})$. We show that if $\mu$ is a polynomial map in one of the two (sets of) variables $\mathbf{x}$ or $\mathbf{y}$, then $G$ is solvable. Moreover, if one knows that $\mu$ is polynomial in one of the variables, the group $G$ is nilpotent if and only if $\mu$ is polynomial in both its variables.
\end{abstract}

\section{CoOrdinate maps, MULTiplication maps AND SIMPLY TRANSITIVE ACTIONS}

In this paper we are concerned with Lie groups $G$ which are diffeomorphic to some space $\mathbb{R}^{n}$. This means that we can define a global chart or coordinate map

$$
\text { co }: G \rightarrow \mathbb{R}^{n}: g \mapsto \operatorname{co}(g) \text {. }
$$

We will always assume that $\operatorname{co}(1)=(0,0,0, \ldots, 0)=\mathbf{0}$. This is no real restriction since we can always use a translation of $\mathbb{R}^{n}$ to adjust a given diffeomorphism to one which satisfies this criterium. Moreover, the reader can verify that this adjustment is of no influence to the polynomiality properties we will be investigating below.

Any coordinate map co $: G \rightarrow \mathbb{R}^{n}$ determines a multiplication map, denoted by $\mu$, which is just the coordinate expression for the multiplication in $G$, so

$$
\mu: \mathbb{R}^{n} \times \mathbb{R}^{n} \rightarrow \mathbb{R}^{n}:(\mathbf{x}, \mathbf{y}) \mapsto \operatorname{co}\left(\operatorname{co}^{-1}(\mathbf{x}) \cdot \operatorname{co}^{-1}(\mathbf{y})\right) .
$$

(Here "." is used to denote the multiplication in G.) We are interested in the case where the multiplication map is polynomial in one or both of its variables. Therefore, we introduce the following definition.

Definition 1.1. Let $f: \mathbb{R}^{n} \times \mathbb{R}^{n} \rightarrow \mathbb{R}^{n}:(\mathbf{x}, \mathbf{y}) \mapsto f(\mathbf{x}, \mathbf{y})$. We say that

(1) $f$ is left-polynomial if $\forall \mathbf{y}_{0} \in \mathbb{R}^{n}$ the map $f_{\mathbf{y}_{0}}: \mathbb{R}^{n} \rightarrow \mathbb{R}^{n}: \mathbf{x} \mapsto f\left(\mathbf{x}, \mathbf{y}_{0}\right)$ is polynomial.

(2) $f$ is right-polynomial if $\forall \mathbf{x}_{0} \in \mathbb{R}^{n}$ the map $f_{\mathbf{x}_{0}}: \mathbb{R}^{n} \rightarrow \mathbb{R}^{n}: \mathbf{y} \mapsto f\left(\mathbf{x}_{0}, \mathbf{y}\right)$ is polynomial.

Received by the editors March 9, 2001 and, in revised form, October 23, 2001.

1991 Mathematics Subject Classification. Primary 22E15.

Key words and phrases. Nilpotent and solvable Lie groups.

This research was conducted while the author was a Postdoctoral Fellow of the Fund for Scientific Research - Flanders (F.W.O.). 
(3) $f$ is one-sided polynomial if $f$ is left- or right-polynomial.

(4) $f$ is bi-polynomial if $f$ is polynomial.

Thus bi-polynomial is just stressing the fact that it is polynomial in all of its variables.

If co: $G \rightarrow \mathbb{R}^{n}$ is a coordinate map, then co also determines a simply transitive (left) action of $G$ on $\mathbb{R}^{n}$ which is given by

$$
\forall g \in G, \forall \mathbf{x} \in \mathbb{R}^{n}:{ }^{g} \mathbf{x}=\operatorname{co}\left(g \cdot \operatorname{co}^{-1}(\mathbf{x})\right)
$$

and conversely, every simply transitive action of $G$ on $\mathbb{R}^{n}$ determines a coordinate map

$$
\text { co }: G \rightarrow \mathbb{R}^{n}: g \mapsto \operatorname{co}(g)={ }^{g} \mathbf{0} .
$$

It is obvious that the constructions (1.2) and (1.3) are each others inverse, from which we deduce that coordinate maps and simply transitive actions are in one-toone correspondence with each other.

For a connected and simply connected nilpotent Lie group $N$, there are two well known coordinate maps, which are both due to Malcev. These maps are called Malcev coordinates of the first resp. second kind and were introduced in 7]. We refer the reader to [7] for the details on the rest of this section.

Let $\mathfrak{n}$ denote the Lie algebra of $N$ and choose a vector space basis $A_{1}, A_{2}, \ldots$, $A_{n}$ of $\mathfrak{n}$ in such a way that $\forall i \in\{1,2, \ldots, n\}$, the space $\mathfrak{n}_{i}$ spanned by $A_{i}, A_{i+1}$, $\ldots, A_{n}$ is an ideal of $\mathfrak{n}$.

It is known that for a simply connected, connected nilpotent Lie group $N$, the exponential map exp $: \mathfrak{n} \rightarrow N$ is a diffeomorphism. So, any element $n \in N$ can be uniquely written in the form $n=\exp \left(\alpha_{1} A_{1}+\alpha_{2} A_{2}+\cdots+\alpha_{n} A_{n}\right)$, with $\alpha_{i} \in \mathbb{R}$. We will refer to the numbers $\left(\alpha_{1}, \alpha_{2}, \ldots, \alpha_{n}\right)$ as the Malcev coordinates of the first kind of $n$. In other words, we have a coordinate map

$$
\text { co }: N \rightarrow \mathbb{R}^{n}: n=\exp \left(\alpha_{1} A_{1}+\alpha_{2} A_{2}+\cdots+\alpha_{n} A_{n}\right) \mapsto \operatorname{co}(n)=\left(\alpha_{1}, \alpha_{2}, \ldots, \alpha_{n}\right) \text {. }
$$

The second coordinate map uses the unique decomposition of an element $n \in N$ into a product $n=\exp \left(\beta_{1} A_{1}\right) \cdot \exp \left(\beta_{2} A_{2}\right) \cdots \cdot \exp \left(\beta_{n} A_{n}\right)$. This decomposition leads to the Malcev coordinates of the second kind:

$$
\begin{aligned}
\mathrm{co}^{\prime}: & N \rightarrow \mathbb{R}^{n}: \\
& n=\exp \left(\beta_{1} A_{1}\right) \cdot \exp \left(\beta_{2} A_{2}\right) \cdots \exp \left(\beta_{n} A_{n}\right) \mapsto \operatorname{co}^{\prime}(n)=\left(\beta_{1}, \beta_{2}, \ldots, \beta_{n}\right) .
\end{aligned}
$$

Theorem 1.2 (Malcev [7]). The multiplication maps induced by the Malcev coordinates of the first and second kind are bi-polynomial.

In this paper we will prove a kind of inverse to this result of Malcev, by showing that the nilpotent Lie groups are the only groups admitting a bi-polynomial multiplication map.

On the other hand, we will also see that any connected and simply connected solvable Lie group admits a coordinate map inducing a left-polynomial (or rightpolynomial) multiplication map, and conversely, that any Lie group admitting a one-sided polynomial multiplication map, is solvable.

\section{Polynomial multiplication maps and solvable Lie groups}

The aim of this section is to prove the following theorem, showing that the class of connected and simply connected solvable Lie groups is exactly the class of Lie groups admitting a one-sided polynomial multiplication map. 
Theorem 2.1. The following three statements are equivalent:

(1) $G$ is a simply connected, connected solvable Lie group.

(2) $G$ admits a coordinate map inducing a left-polynomial multiplication map.

(3) G admits a coordinate map inducing a right-polynomial multiplication map.

We will prove this theorem in a few steps. First, we show the equivalence of the last 2 statements.

Lemma 2.2. Let $G$ be a Lie group. Then

$G$ admits a coordinate map inducing a left-polynomial multiplication map

$\mathbb{1}$

$G$ admits a coordinate map inducing a right-polynomial multiplication map.

Proof. Let co $: G \rightarrow \mathbb{R}^{n}$ be a coordinate map inducing a multiplication map $\mu$. We define a second coordinate map

$$
\operatorname{co}^{\prime}: G \rightarrow \mathbb{R}^{n}: g \mapsto \operatorname{co}^{\prime}(g)=\operatorname{co}\left(g^{-1}\right)
$$

inducing a multiplication map $\mu^{\prime}$. We have for all $\mathbf{x}, \mathbf{y} \in \mathbb{R}^{n}$ :

$$
\begin{aligned}
\mu^{\prime}(\mathbf{x}, \mathbf{y}) & =\operatorname{co}^{\prime}\left(\operatorname{co}^{-1}(\mathbf{x}) \cdot \operatorname{co}^{-1}(\mathbf{y})\right) \\
& =\operatorname{co}\left(\left(\operatorname{co}^{-1}(\mathbf{x}) \cdot \operatorname{co}^{\prime-1}(\mathbf{y})\right)^{-1}\right) \\
& =\operatorname{co}\left(\operatorname{co}^{-1}(\mathbf{y}) \cdot \operatorname{co}^{-1}(\mathbf{x})\right) \quad\left(\operatorname{use~co}^{-1}(\mathbf{x})=\left(\operatorname{co}^{-1}(\mathbf{x})\right)^{-1}\right) \\
& =\mu(\mathbf{y}, \mathbf{x}) .
\end{aligned}
$$

This shows that $\mu$ is left- (resp. right-) polynomial if and only if $\mu^{\prime}$ is right- (resp. left-) polynomial, which proves the lemma.

The next step in our investigation is the construction of a faithful linear representation for each Lie group admitting a coordinate map inducing a one-sided polynomial multiplication map. The idea for such a representation was introduced in 3 . We retake this idea here and adjust it to fit our purposes.

Proposition 2.3. Let $G$ be a Lie group admitting a coordinate map co $: G \rightarrow \mathbb{R}^{n}$, such that the induced multiplication map $\mu$ is one-sided polynomial. Then $G$ admits a faithful linear representation

$$
\rho: G \rightarrow \operatorname{Gl}(m, \mathbb{R}), \text { for some positive integer } m .
$$

Moreover, in case $\mu$ is bi-polynomial, the presentation $\rho$ can be taken in such a way that the entries of $\rho(g)$ depend polynomially on $\operatorname{co}(g)$.

Proof. By Lemma 2.2 we may assume that $\mu$ is right-polynomial. This means that $G$ acts simply transitively on $\mathbb{R}^{n}$, in such a way that the action of any element $g \in G$ is expressed by a polynomial map. In other words, we have a faithful representation

$$
\varphi: G \rightarrow \mathrm{P}\left(\mathbb{R}^{n}\right),
$$

where $\mathrm{P}\left(\mathbb{R}^{n}\right)$ (the group of polynomial diffeomorphisms) is used to denote the group of bijections $p: \mathbb{R}^{n} \rightarrow \mathbb{R}^{n}$ such that both $p$ and $p^{-1}$ are polynomial maps. In [6] Theorem 4.2] we showed that there exists a positive integer $d$ such that the degree of $\varphi(g)$ is less than $d$, for all $g \in G$. Now, let $\mathrm{P}\left(\mathbb{R}^{n}, \mathbb{R}\right)$ denote the vector space 
of all polynomial maps from $\mathbb{R}^{n}$ to $\mathbb{R}$. This vector space can be made into a right $G$-module by defining

$$
\forall g \in G, \forall p \in \mathrm{P}\left(\mathbb{R}^{n}, \mathbb{R}\right): p^{g}=p \circ \varphi(g) .
$$

Now, let $V$ be the $\mathbb{R} G$-submodule of $\mathrm{P}\left(\mathbb{R}^{n}, \mathbb{R}\right)$ generated by the set of linear maps $\left\{p_{1}, p_{2}, \ldots, p_{n}\right\} \subseteq \mathrm{P}\left(\mathbb{R}^{n}, \mathbb{R}\right)$, where

$$
p_{i}: \mathbb{R}^{n} \rightarrow \mathbb{R}:\left(x_{1}, x_{2}, \ldots, x_{n}\right) \mapsto x_{i} .
$$

Due to the existence of the bound $d$ on the degrees of $\varphi(G)$, the space $V$ is a finitedimensional subspace of $\mathrm{P}\left(\mathbb{R}^{n}, \mathbb{R}\right)$. Therefore, we can fix a basis of $V$ and identify $V$ with some space $\mathbb{R}^{m}$.

If we denote this identification with id $: V \rightarrow \mathbb{R}^{m}$, we obtain a map

$$
\rho: G \rightarrow \operatorname{Gl}(m, \mathbb{R}) \text {, defined by } \operatorname{id}(p) \rho(g)=\operatorname{id}\left(p^{g}\right)=\operatorname{id}(p \circ \varphi(g)), \quad \forall p \in V .
$$

(Here $\operatorname{id}(p) \rho(g)$ denotes multiplication of a matrix with a row-vector.) It is now easy to verify that this map $\rho$ is a faithful representation and that the entries of $\rho(g)$ depend polynomially on $\operatorname{co}(g)$, in case $\mu$ is bi-polynomial.

We are now ready to prove the main result of this section.

Proof of Theorem [2.1. We have already seen that the last two statements are equivalent.

If $G$ admits a coordinate map co : $G \rightarrow \mathbb{R}^{n}$, inducing a one-sided multiplication map, then $G$ admits a faithful linear representation. However, it is known that all Lie groups $G$ which are homeomorphic to $\mathbb{R}^{n}$ and which admit a faithful linear representation are solvable (the reader can consult [6. Proposition 5.1] for details on this claim). So, this proves that (2) or (3) implies (1).

Conversely, if $G$ is a connected and simply connected solvable Lie group, then $G$ admits a simply transitive action on some space $\mathbb{R}^{n}$, which is polynomial (i.e. the action of any element $g \in G$ is expressed by a polynomial map). (See [4] or [5] for the construction of such actions.) As was already indicated in the first section of this paper, such a simply transitive action is equivalent to a coordinate map co : $G \rightarrow \mathbb{R}^{n}$, which in this case induces a right-polynomial multiplication map $\mu$. This shows that (1) implies (3), which finishes the proof.

By interpreting a coordinate map as a simply transitive action, we find the following result, which is a generalization of a result of L. Auslander ([2]) on affine actions. (It also generalizes [6] Theorem 5.2].)

Corollary 2.4. Let $G$ be a Lie group acting simply transitively and via polynomial maps on $\mathbb{R}^{n}$. Then $G$ is solvable.

Remark 2.5. A key ingredient to obtain this result was the use of [6, Theorem 4.2] (via Proposition 2.3) showing that any polynomial action on $\mathbb{R}^{n}$ of a connected Lie group is of bounded degree. It seems impossible to avoid this kind of result in proving the theorem above.

\section{Polynomial multiplication maps and nilpotent Lie groups}

If $G$ is an abelian Lie group with a one-sided polynomial multiplication map, then obviously this multiplication map has to be bi-polynomial. This is however not the only class of groups for which this result holds. Indeed, it turns out that 
the class satisfying the property that "one-sided polynomial $\Rightarrow$ bi-polynomial" is exactly the class of nilpotent groups. This is the context of the following theorem.

Theorem 3.1. Let $G$ be a Lie group admitting a coordinate map co $: G \rightarrow \mathbb{R}^{n}$, inducing a one-sided polynomial multiplication map $\mu$. Then

$G$ is nilpotent $\Leftrightarrow \mu$ is bi-polynomial.

Proof. Assume that $G$ is nilpotent. By Lemma 2.2, we may suppose that $\mu$ is right-polynomial, and therefore, co determines a polynomial and simply transitive action of $G$ on $\mathbb{R}^{n}$. Lemma 3.1(c) of 3 says exactly that there exists a polynomial bijection $p: \mathbb{R}^{n} \rightarrow \mathbb{R}^{n}$ with polynomial inverse $p^{-1}$ such that

$$
\forall g \in G: p(\operatorname{co}(g))=\operatorname{co}^{\prime}(g) \text {, }
$$

where $\operatorname{co}^{\prime}(g)$ denote the Malcev coordinates of the first (or second) kind. If we use $\mu^{\prime}$ to denote the (bi-polynomial) multiplication map corresponding to these Malcev coordinates, we have that

$$
\begin{aligned}
\mu(\mathbf{x}, \mathbf{y}) & =\operatorname{co}\left(\mathrm{co}^{-1}(\mathbf{x}) \cdot \mathrm{co}^{-1}(\mathbf{y})\right) \\
& =p^{-1} \operatorname{co}^{\prime}\left(\operatorname{co}^{\prime-1}(p(\mathbf{x})) \cdot \operatorname{co}^{-1}(p(\mathbf{y}))\right) \\
& =p^{-1} \mu^{\prime}(p(\mathbf{x}), p(\mathbf{y})) .
\end{aligned}
$$

This last expression is clearly bi-polynomial.

Now, suppose that the multiplication map $\mu$ is bi-polynomial. We can use this map to define a complex Lie group $G_{\mathbb{C}}$, with underlying set $\mathbb{C}^{n}$ and where the multiplication is given by the same polynomial map $\mu$, now defined on $\mathbb{C}^{n}$ :

$$
\mathbb{C}^{n} \times \mathbb{C}^{n} \rightarrow \mathbb{C}^{n}:\left(\mathbf{z}_{1}, \mathbf{z}_{2}\right) \mapsto \mu\left(\mathbf{z}_{1}, \mathbf{z}_{2}\right) .
$$

Note that we can use Theorem 2.1 to conclude that $G_{\mathbb{C}}$ is solvable. $G_{\mathbb{C}}$ contains a copy of $G$ via the embedding co: $G \rightarrow \mathbb{R}^{n} \subseteq \mathbb{C}^{n}=G_{\mathbb{C}}$.

Although we proved Proposition 2.3 only for real Lie groups, it is straightforward to generalize it to the complex case and to deduce that there exists a faithful linear representation

$$
\rho: G_{\mathbb{C}} \rightarrow \mathrm{Gl}(m, \mathbb{C}): \mathbf{z} \mapsto \rho(\mathbf{z})
$$

where $\rho(\mathbf{z})$ depends polynomially on the components of $\mathbf{z}$.

Moreover, as $G$ is connected and solvable, we may suppose that the representation is in uppertriangular form (Lie's Theorem).

Now, an element on the main diagonal must never be zero, however, this is only true when this entry, which is polynomial in $\mathbf{z} \in \mathbb{C}^{n}$, is in fact a constant polynomial (by Hilbert's Nullstellensatz). Therefore, the entries on the main diagonal should all be equal to 1 . This shows that $\rho\left(G_{\mathbb{C}}\right)$ is a unipotent group, from which we may conclude that $G_{\mathbb{C}}$, and so also $G$, is a nilpotent group.

Note. One direction of this theorem is mentioned without proof in a paper of L. Auslander ([1, page 230]) and is there credited to R. Tolimieri. However, there is no track of a proof of this result in literature.

\section{REFERENCES}

[1] Auslander, L. An exposition of the structure of solvmanifolds. Part I: Algebraic Theory. Bull. Amer. Math. Soc., 1973, 79 2, pp. 227-261. MR 58:6066a

[2] Auslander, L. Simply Transitive Groups of Affine Motions. Amer. J. Math., 1977, 99 (4), pp. 809-826. MR 56:5782 
[3] Benoist, Y. and Dekimpe, K. The Uniqueness of Polynomial Crystallographic Actions. Math. Ann., 2002, 322, pp. 563-571.

[4] Dekimpe, K. Semi-simple splittings for solvable Lie groups and polynomial structures. Forum Math., 2000, 12, pp. 77-96. MR 2001h:22009

[5] Dekimpe, K. Solvable Lie algebras, Lie groups and polynomial structures. Compositio Mathematica, 2000, 121, pp. 183-204. MR 2001e:17014

[6] Dekimpe, K. and Igodt, P. Polynomial Alternatives for the Group of Affine Motions. Math. Zeit., 2000, 234, pp. 457-485. MR 2001k:57042

[7] Malcev, A. I. On a class of homogeneous spaces. Translations A.M.S., 1951, 39, pp. 1-33. MR 12:589e

Katholieke Universiteit Leuven, Campus Kortrijk, B-8500 Kortrijk, Belgium

E-mail address: Karel.Dekimpe@kulak.ac.be 